\title{
Surgical Resection for Pulmonary Metastasis from Pancreatic and Biliary Tract Cancer
}

\author{
TETSUZO TAGAWA $^{1,2}$, KENSAKU ITO $^{1}$, KENGO FUKUZAWA $^{3}$, TATSURO OKAMOTO ${ }^{2}$, \\ ATSURO YOSHIMURA ${ }^{3}$, TAKAHIDE KAWASAKI ${ }^{3}$, TAKASHI MASUDA ${ }^{3}$, \\ KENTARO IWAKI $^{3}$, TAKAHIRO TERASHI ${ }^{3}$, MASAHIRO OKAMOTO ${ }^{3}$, \\ AKIO SHIROMIZU $^{3}$, AKIRA MOTOHIRO ${ }^{1}$ and YOSHIHIKO MAEHARA ${ }^{2}$ \\ Departments of ${ }^{1}$ General Thoracic Surgery, and ${ }^{3}$ Surgery, Oita Red Cross Hospital, Oita, Japan; \\ ${ }^{2}$ Department of Surgery and Science, Graduate School of Medical Sciences, \\ Kyushu University, Fukuoka, Japan
}

\begin{abstract}
Aim: To determine the efficacy of pulmonary metastasectomy for pancreatic and biliary tract cancer. Patients and Methods: Ten patients who underwent therapeutic pulmonary metastasectomy after resection for pancreatic and biliary tract cancer at our Institution from 2006 to 2016 were retrospectively evaluated. Results: The primary site was the pancreas in four patients and biliary tract in six. Nine patients had single metastasis, and one patient had bilateral multiple metastases. The median time from surgery for the primary tumor to pulmonary resection was 23.3 months (range $=0-47.1$ months). One patient underwent lobectomy, while nine patients underwent partial resection. One patient had incomplete resection due to pleural dissemination. There were no postoperative mortalities or major morbidities. The mean follow-up period was 26.0 months. The median survival time was 38.5 months, and the estimated 5-year overall survival was $38.9 \%$ after pulmonary resection. Five patients had recurrent disease after pulmonary resection, with a median recurrence-free interval of 6.0 months. One patient underwent second pulmonary resection for a solitary lung recurrence. Conclusion: Despite the poor prognoses of these cancer types, pulmonary metastasectomy can significantly prolong survival in selected patients with pancreatic and biliary tract cancer.
\end{abstract}

Pancreatic and biliary tract cancer are highly aggressive malignancies with an extremely poor prognosis. Despite the

Correspondence to: Tetsuzo Tagawa, Department of General Thoracic Surgery, Oita Red Cross Hospital, 3-2-37, Chiyo-machi, Oita, 870-0033, Japan. Tel: +81 975326181, Fax: +81 975331207, e-mail: ttagawa@surg2.med.kyushu-u.ac.jp

Key Words: Lung surgery, metastatic lung tumor, pancreatic cancer, biliary tract cancer. recent progress of surgical techniques and chemotherapy, the 5 -year survival rate is less than $5 \%$ for patients with pancreatic cancer and $5-15 \%$ for those with biliary tract cancer $(1,2)$. While surgical resection offers the only potentially curative treatment, surgical candidates account for fewer than $20 \%$ of cases of pancreatic cancer and fewer than $35 \%$ of cases of biliary tract cancer, and the prognosis is unsatisfactory even after complete resection $(2,3)$. Among patients who have undergone surgical resection, the lung is a major site of first recurrence in both types of cancer, and some patients have only a solitary lung metastasis (4-6).

Pulmonary metastasectomy is a widely recognized viable treatment strategy for various solid-organ malignancies. According to the report from the International Registry of Lung Metastases, the actuarial 5-year survival rate for various histologies combined after complete pulmonary metastasectomy was $36 \%$ (7). However, since there have been very few studies concerning pulmonary metastasectomy in patients with pancreatic and biliary tract cancer, the benefit is still controversial $(6,8)$.

We retrospectively reviewed our institutional experience and analyzed the outcomes of 10 patients who underwent surgery for pulmonary metastases of pancreatic and biliary tract cancer.

\section{Patients and Methods}

We retrospectively reviewed the clinical records of 10 patients who underwent pulmonary metastasectomy after curative-intent resection of pancreatic and biliary tract cancer at our Institution from January 2006 to September 2016. These patients accounted for $2.1 \%$ (10 out of 478) of all patients who underwent curative-intent surgery for pancreatic and biliary tract cancer during the same period at our Institution.

Newly-identified pulmonary non-spiculated nodules without ground-glass opacity by chest computed tomography (CT) after surgery for the primary tumor were followed up by CT for several 
Table I. Patient characteristics with regard to primary tumor.

\begin{tabular}{|c|c|c|c|c|c|c|c|c|}
\hline Patient & $\begin{array}{l}\text { Age, years/ } \\
\text { gender }\end{array}$ & $\begin{array}{l}\text { Primary } \\
\text { site }\end{array}$ & Stage & $\begin{array}{l}\text { Induction } \\
\text { therapy }\end{array}$ & Procedure & $\begin{array}{l}\text { Complete } \\
\text { resection }\end{array}$ & $\begin{array}{c}\text { Postoperative } \\
\text { therapy }\end{array}$ & $\begin{array}{c}\text { Preoperative } \\
\text { CA19-9 (U/ml) }\end{array}$ \\
\hline 1 & $63 / \mathrm{M}$ & Panc & T3N1M0 (IIB) & None & PD & Yes & Gem & Unknown \\
\hline 2 & $73 / \mathrm{F}$ & Panc & T3N0M0 (IIA) & CRT(Gem) & PD & Yes & Gem & Unknown \\
\hline 3 & $66 / \mathrm{M}$ & Panc & T3N1M0 (IIB) & None & DP & Yes & Gem & Unknown \\
\hline 4 & $82 / \mathrm{F}$ & Panc & T3N1M0 (IIB) & None & DP & No & Gem & 738 \\
\hline 5 & $78 / \mathrm{F}$ & EHB & T2N0M0 (IB) & None & Hep & Yes & $\mathrm{RT}$ & 65 \\
\hline 6 & $68 / \mathrm{M}$ & IHB & T3N1M0 (IIB) & None & Hep & Yes & Gem & 4882 \\
\hline 7 & $71 / \mathrm{M}$ & GB & T3N1M0 (IIIB) & GFP & Hep & Yes & GFP & 37788 \\
\hline 8 & $68 / \mathrm{M}$ & EHB & T4N1M0 (IVA) & None & Hep & No & Gem & 22529 \\
\hline 9 & $80 / \mathrm{M}$ & IHB & T4N0M1 (IV) & None & Hep & No & Gem & 6 \\
\hline 10 & $87 / \mathrm{M}$ & GB & T4N2M0 (IVB) & GFP & HPD & Yes & None & 403 \\
\hline
\end{tabular}

M: Male; F: female; Panc: pancreas; EHB: extrahepatic bile duct; IHB: intrahepatic bile duct; GB: gallbladder; CRT: chemoradiotherapy; Gem: gemcitabine; GFP: gemcitabine+5-fluorouracil+cisplatin; PD: pancreaticoduodenectomy; DP: distal pancreatectomy; Hep: hepatectomy; HPD: hepatopancreaticoduodenectomy; RT: radiotherapy.

Table II. Patient characteristics with regard to tumor recurrence.

\begin{tabular}{|c|c|c|c|c|c|c|c|c|c|}
\hline Patient & $\begin{array}{c}\text { DFI } \\
\text { (months) }\end{array}$ & $\begin{array}{c}\text { Preop CA19-9 } \\
(\mathrm{U} / \mathrm{ml})\end{array}$ & $\begin{array}{l}\text { Tumor } \\
\text { number }\end{array}$ & $\begin{array}{l}\text { Maximum lung } \\
\text { tumor size (mm) }\end{array}$ & Procedure & $\begin{array}{l}\text { Complete } \\
\text { resection }\end{array}$ & $\begin{array}{c}\text { Postoperative } \\
\text { therapy }\end{array}$ & $\begin{array}{l}\text { First recurrence } \\
\text { after mtx }\end{array}$ & $\begin{array}{c}\text { Prognosis, } \\
\text { months/status }\end{array}$ \\
\hline 1 & 27.7 & 15 & 1 & 10 & VATS wedge & Yes & S-1 & Lung & 39/DOD \\
\hline 2 & 47.1 & 32 & 1 & 15 & VATS wedge & Yes & Gem & Lung & 65/DD \\
\hline 3 & 30.8 & 8 & 1 & 5 & Open wedge & Yes & GemNab & Lung, PLD & $37 / \mathrm{AD}$ \\
\hline 4 & 20.8 & 49 & 1 & 14 & VATS wedge & Yes & None & Bone, PLD & $10 / \mathrm{DD}$ \\
\hline 5 & 7.9 & Unknown & 1 & 30 & Open wedge & Yes & None & None & 24/DD \\
\hline 6 & 0.0 & 9 & 1 & 12 & VATS wedge & Yes & S-1 & Lung & 16/DD \\
\hline 7 & 40.5 & 67 & 1 & 8 & Open wedge & Yes & None & None & 3/ANED \\
\hline 8 & 14.5 & 12 & 1 & 29 & Open lobectomy & Yes & None & None & 51/ANED \\
\hline 9 & 16.1 & 2 & 1 & 10 & VATS wedge & No & Gem & N/A & 14/AD \\
\hline 10 & 25.7 & 5 & 4 & 12 & Bil. VATS wedge & Yes & None & None & 3/ANED \\
\hline
\end{tabular}

DFI: Disease-free interval; VATS: video-assisted thoracic surgery; Bil: bilateral; CT: chemotherapy; Gem: gemcitabine; GemNab: gemcitabine+nabpaclitaxel; mtx: lung metastasectomy; PLD: pleural dissemination; N/A: not applicable; DOD: dead of other disease; DD: dead of disease; AD: alive with disease; ANED: alive with no evidence of disease.

months. The enlarged nodules were suspected as lung metastases. Only one patient had synchronous pulmonary metastasis, which was initially considered an inflammatory nodule but then increased in size after primary site surgery. All patients underwent full restaging by contrast-enhanced thoracic and abdominal $\mathrm{CT}$, isotonic bone scanning, and sometimes $\left[{ }^{18} \mathrm{~F}\right]$-positron-emission tomography to exclude extrapulmonary metastasis. A multidisciplinary team including thoracic surgeons, hepatobiliary surgeons, medical oncologists, and radiation oncologists evaluated each patient and decided to proceed with pulmonary resection if the patient had no metastatic disease at another site and complete resection was deemed likely to be achieved regardless of the number of lesions. Multiple tumors in the bilateral lungs were not considered to be a contraindication. One patient (patient 2) developed an additional lung metastasis after pulmonary resection, and further resection was performed. Regarding the surgical procedure, minimal lung resection with sufficient surgical margin from the tumors, preferably wedge resection, was principally selected.
The statistical data analysis was performed using the JMP software package, version 5.0 (SAS Institute, Inc., Cary, NC, USA). The disease-free interval (DFI) was defined as the period between curative primary site resection and the first detection of pulmonary nodules. The survival and recurrence-free interval were calculated from the date of the surgery until the date of death or the appearance of recurrent disease. The survival rates were calculated using the Kaplan-Meier method.

\section{Results}

The patients' clinical characteristics are shown in Tables I and II. Six patients were male, and four were female, and the average age was 71.6 years (range $=63-82$ years). Two patients (patients 7 and 10) had received induction chemotherapy, and one patient (patient 2) had received induction chemoradiotherapy before primary site surgery. 


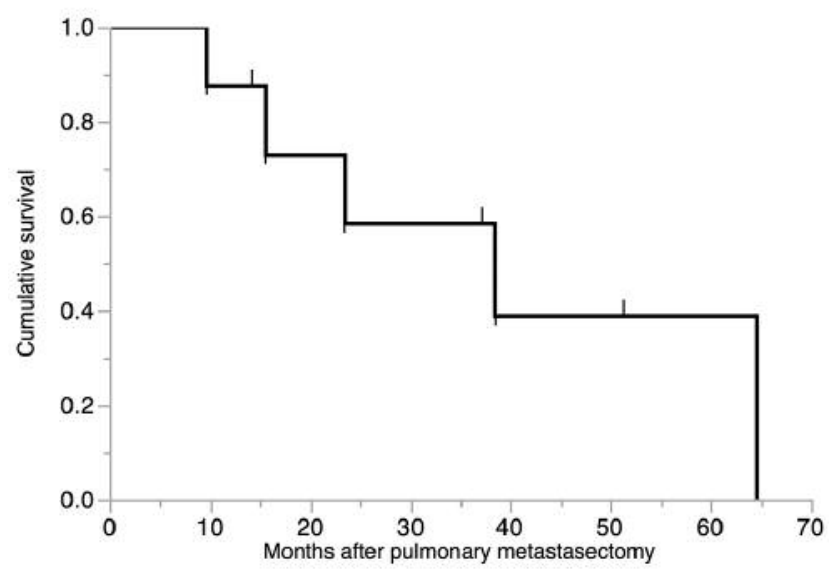

Figure 1. Overall survival of 10 patients after pulmonary metastasectomy. The median survival time was 38.5 months, and 5-year overall survival was $38.9 \%$.

The primary tumor had been completely resected in 7 out of 10 patients. Two patients (patients 4 and 8) underwent R1 resection, which is defined as grossly complete resection with microscopically positive margins. These two patients received chemotherapy after surgery and no recurrence was found until the pulmonary metastasis was revealed. One patient (patient 9) underwent $\mathrm{R} 2$ resection, which is defined as gross residual disease, due to a peritoneal nodule which proved to be a disseminated nodule afterward. This patient had chemotherapy after hepatectomy, and no recurrence other than pulmonary metastasis was evident. Eight patients had received chemotherapy after surgery, and one (patient 5) had received adjuvant radiotherapy. The DFI ranged from 0 to 47.1 months, with a median of 23.3 months. In all patients, pulmonary metastasis was the first recurrent site after primary site surgery. The serum carbohydrate antigen 19-9 (CA19-9) level was elevated in six patients before primary site surgery and in two patients before pulmonary resection. All but one patient achieved complete resection. One patient (patient 9) had pleural dissemination that was identified during pulmonary resection. Another patient (patient 10) had bilateral multiple metastases that were completely resected by a bilateral video-assisted thoracic surgery procedure. There were no postoperative deaths or severe postoperative complications. Five patients underwent adjuvant chemotherapy after pulmonary resection.

Five out of nine patients (excluding one patient with incomplete resection) experienced recurrence after pulmonary resection, with a median recurrence-free interval of 6.0 months. The pattern of initial recurrence was lung metastasis in three patients, lung metastasis with pleural dissemination in one patient, and bone metastasis with pleural dissemination in one patient. One patient (patient 2) underwent additional pulmonary resection for solitary lung metastasis 23 months after the first pulmonary resection.

The mean follow-up period was 26.0 months. At the time of analysis, five patients had died, two patients were alive with disease and three patients were alive without disease. The median survival time after pulmonary metastasectomy was 38.5 months, and the estimated 5-year overall survival was $38.9 \%$ (Figure 1). The median survival time after primary site surgery was 66.2 months, and the estimated 5year overall survival was $64.3 \%$. In a univariate analysis, no factors, including the DFI and serum CA19-9 level before lung resection, were predictive of the survival.

\section{Discussion}

In the present study, we showed that select patients could expect long-term survival after pulmonary metastasectomy for pancreatic and biliary tract cancer. Pulmonary metastasectomy for various solid-organ malignancies has been reported as an efficacious treatment strategy potentially associated with long-term survival: however, few studies have reported such results with pulmonary metastasectomy for pancreatic and biliary tract cancer, probably due to their extremely poor outcomes. However, some patients experience distant metastasis limited to the lung after resection of pancreatic and biliary tract cancer, whom might benefit from metastasectomy. Arnaoutakis et al. reported the results of nine patients who underwent pulmonary metastasectomy for pancreatic cancer (6). They showed that pulmonary metastasectomy can be performed safely and may be a beneficial procedure, with an encouraging median overall survival of 52 months. They also found a worse median overall survival of 23 months in the patients who also had isolated pulmonary metastasis but did not undergo metastasectomy. Although the difference in the survival between the two groups may be attributed to selection bias, their report indicated that long-term survival could be achieved by pulmonary metastasectomy in select patients with pancreatic cancer.

Robinson et al. recently reported the results of pulmonary resections in patients with pancreaticobiliary cancer (8). Out of 29 patients who received therapeutic lung resection after definitive treatment of pancreaticobiliary cancer, 16 underwent resection for pulmonary metastasis, 12 for primary lung cancer, and one for granuloma. According to their report, the patients who underwent pulmonary metastasectomy after pancreatic resection accounted for approximately $1.4 \%$ of all patients who had undergone pancreatic resection in their institution in the study period, while the ratio was $2.1 \%$ in the present study. These results obviously indicate the rarity of candidates for pulmonary metastasectomy from pancreaticobiliary cancer. The median survival time and estimated 5-year survival rate of those who 
underwent pulmonary metastasectomy was 28 months and $37 \%$, respectively, in their report. Their survival results were largely consistent with the present study, indicating that longterm survival could be achieved in highly select patients. They also reported that the serum CA19-9 level before lung resection was an independent predictor of survival. In the present study, only two out of nine patients had an elevated serum CA19-9 level before lung resection, and it was not predictive of survival. Although CA19-9 is reported to be an independent predictor of the outcome in patients who undergo resection of pancreatic cancer, its role in pulmonary metastasectomy should be investigated further (9).

The report from the International Registry of Lung Metastases found that the primary tumor type, DFI, and the number of metastases were highly significant prognostic factors (7). In the present study, the DFI ranged from 0 to 47 months, with a median of 23.3 months, which was not predictive of survival. Some patients with a relatively short DFI (<18 months) survived more than 24 months after pulmonary metastasectomy. Robinson et al. also found in their study that some patients with a short DFI ( $<12$ months) survived relatively long-term (8). Wangjam et al. reported that the DFI of the patients who experienced recurrence only in the lung after curative resection of pancreatic cancer was longer than in those who experienced recurrence in other sites, such as the liver and peritoneum (10). They also reported that pancreatic cancer with the first recurrence only in the lung was associated with a significantly better outcome than those with recurrences in other sites. They speculated that pancreatic cancer with different sites of recurrence might have different biological background. In terms of the number of metastases, eight out of nine patients with complete pulmonary metastasectomy had a solitary lung metastasis in the present study. The favorable outcome in the present study may be attributed to this fact, since resectability and number of metastases were two constitutive factors of prognostic grouping developed by the International Registry of Lung Metastases (7). Since patient selection is crucial for making pulmonary metastasectomy a meaningful strategy in these intractable cancer types with few patients, the impact of the DFI and number of metastases on the survival should be investigated further.

One limitation associated with the present study is the small population, as we only included 10 patients from a single institution, and the retrospective nature of the study. In addition, the treatment strategy and the use of chemotherapeutic drugs have varied over time. Therefore, the results should be interpreted with caution.

In conclusion, despite the very small number of patients in this study, we found that pulmonary metastasectomy for pancreatic and biliary tract cancer can significantly prolong survival in highly selected patients.

\section{References}

1 Hidalgo M: Pancreatic cancer. N Engl J Med 362: 1605-1617, 2010.

2 Horgan AM, Amir E, Walter T and Knox JJ: Adjuvant therapy in the treatment of biliary tract cancer: a systematic review and meta-analysis. J Clin Oncol 30: 1934-1940, 2012.

3 Garrido-Laguna I and Hidalgo M: Pancreatic cancer: from stateof-the-art treatments to promising novel therapies. Nat Rev Clin Oncol 12: 319-334, 2015.

4 Katz MHG, Wang H, Fleming JB, Sun CC, Hwang RF, Wolff RA, Varadhachary G, Abbruzzese JL, Crane CH, Krishnan S, Vauthey J-N, Abdalla EK, Lee JE, Pisters PWT and Evans DB: Long-term survival after multidisciplinary management of resected pancreatic adenocarcinoma. Ann Surg Oncol 16: 836$847,2009$.

5 Jarnagin WR, Ruo L, Little SA, Klimstra D, D'Angelica M, DeMatteo RP, Wagman R, Blumgart LH and Fong Y: Patterns of initial disease recurrence after resection of gallbladder carcinoma and hilar cholangiocarcinoma: implications for adjuvant therapeutic strategies. Cancer 98: 1689-1700, 2003.

6 Arnaoutakis GJ, Rangachari D, Laheru DA, Iacobuzio-Donahue CA, Hruban RH, Herman JM, Edil BH, Pawlik TM, Schulick RD, Cameron JL, Meneshian A, Yang SC and Wolfgang CL: Pulmonary resection for isolated pancreatic adenocarcinoma metastasis: an analysis of outcomes and survival. J Gastrointest Surg 15: 1611-1617, 2011.

7 Pastorino U, Buyse M, Friedel G, Ginsberg RJ, Girard P, Goldstraw P, Johnston M, McCormack P, Pass H and Putnam JB: Long-term results of lung metastasectomy: Prognostic analyses based on 5206 cases. J Thorac Cardiovasc Surg 113: 37-49, 1997.

8 Robinson LA, Tanvetyanon T, Springett G, Fontaine J, Toloza E, Hodul P, Pimiento JM and Malafa M: Pulmonary metastasectomy for suspected pancreaticobiliary cancer. J Thorac Cardiovasc Surg 152: 75-82, 2016.

9 Marchegiani G, Andrianello S, Malleo G, De Gregorio L, Scarpa A, Mino-Kenudson M, Maggino L, Ferrone CR, Lillemoe KD, Bassi C, Castillo CF-D and Salvia R: Does size matter in pancreatic cancer? Reappraisal of tumour dimension as a predictor of outcome beyond the TNM. Ann Surg, 2016. [Epub ahead of print]

10 Wangjam T, Zhang Z, Zhou XC, Lyer L, Faisal F, Soares KC, Fishman E, Hruban RH, Herman JM, Laheru D, Weiss M, Li M, De Jesus-Acosta A, Wolfgang CL and Zheng L: Resected pancreatic ductal adenocarcinomas with recurrence limited in lung have a significantly better prognosis than those with other recurrence patterns. Oncotarget 6: 36903-36910, 2015.

Received January 17, 2017

Revised February 25, 2017 Accepted February 25, 2017 